\title{
Calf muscle activity alteration with foot orthoses insertion during walking measured by fine-wire electromyography
}

\author{
Hiroshi Akuzawa, PT, MS'1), Atsushi Imai, PhD ${ }^{2)}$, Satoshi Iizuka, MS ${ }^{1)}$, \\ NaOto Matsunaga, $\mathrm{MS}^{1)}$, Koji KaneOKa, PhD, $\left.\mathrm{MD}^{2}\right)^{*}$ \\ 1) School of Sport Sciences, Waseda University, Japan \\ 2) Faculty of Sport Sciences, Waseda University: 2-579-15, Mikajima, Tokorozawa, Saitama, Japan
}

\begin{abstract}
Purpose] The purpose of the study was to assess the muscle activity change of the tibialis posterior, flexor digitorum longus, and peroneus longus during gait with orthoses. [Subjects and Methods] Sixteen healthy males participated in this study. Activity of each muscle was measured by using fine-wire and surface electromyography. Gait task was performed by the participants barefoot, with footwear and with orthoses. The electromyography data from a stance phase of each gait trial were used for analysis. The stance phase was divided into contact, midstance, and propulsion phases. The data from ten participants were extracted for final analysis, as electromyography measurements were unsuccessful for the other six. [Results] The results demonstrated that orthoses significantly reduced the tibialis posterior muscle activity in the propulsion phase compared to that in the barefoot condition. Although there was a significant difference in the midstance phase, post hoc analysis did not indicate significant differences among the phases. No significant electromyography amplitude change was detected in flexor digitorum longus and peroneus longus. [Conclusion] Orthothes reduced the tibialis posterior activity level during gait. This result may be beneficial for patients with injuries related to excessive activity of tibialis posterior. Key words: Tibialis posterior, Flexor digitorum longus, Insole
\end{abstract}

(This article was submitted Jul. 27, 2016, and was accepted Sep. 7, 2016)

\section{INTRODUCTION}

Foot orthoses is often used to correct the foot alignment for the treatment or prevention of the injuries and for performance enhancement. Clinically, patients who have foot as well as knee and hip joint problems use foot orthoses to alleviate symptoms. In sports, foot orthoses is sometimes prescribed for improving propulsion force transmission, absorbing ground reaction force, and increasing or decreasing muscle activity level. The effect of orthoses has been researched and recent systematic review regarding orthoses reported that kinematic, shock attenuation, and neuromuscular paradigms are the main effects of orthoses ${ }^{1}$. Specifically, kinematic changes led by orthotic use have been reported in numerous studies. These studies described that foot eversion angle and moment are reduced by orthoses ${ }^{2,3)}$. In recent years, the number of studies reporting the effect of orthoses on neuromuscular paradigms has been increasing.

Posterior calf muscles are fundamental in producing propulsion force during walking. Tibiails posterior (TP), peroneus longus (PL) and flexor digitorum longus (FDL) produce a torque toward plantar flexion, which functions as propulsion force during walking. In addition to this, these muscles control foot pronation or supination and maintain the arches of the foot ${ }^{4-6)}$. These functions can change the foot rigidity and flexibility that adapt the mechanical demands on the foot. Orthoses are considered to compensate the function of the calf muscles and alter the muscle activity pattern. Several studies researched the effect of the orthoses on the TP, PL, and gastrocnemius muscle ${ }^{7-9)}$. The results of those studies showed that the muscle

*Corresponding author. Koji Kaneoka (E-mail: kaneoka@waseda.jp)

(C2016 The Society of Physical Therapy Science. Published by IPEC Inc.

This is an open-access article distributed under the terms of the Creative Commons Attribution Non-Commercial No Derivatives (by-nc-nd) License $<$ http://creativecommons.org/licenses/by-nc-nd/4.0/>. 
activity level of TP decreased, while the PL demonstrated increased muscle activity level during walking. However, no study has researched the effect of orthoses on the muscle activity level of the TP, PL, and FDL simultaneously.

Therefore, the purpose of this study was to determine the TP, FDL, and PL muscle activity change resulting from orthotic insertion during the walking stance phase by using a fine-wire and a surface electromyography (EMG). Moreover, stance phase was divided into three phases and muscle activity alteration of each muscle was analyzed in each phase.

\section{SUBJECTS AND METHODS}

Sixteen healthy male students (age: $24 \pm 4.9$ years, height: $168.4 \pm 5.1 \mathrm{~cm}$, and weight: $60.9 \pm 7.8 \mathrm{~kg}$ ) participated in this study. Participants who had injuries or surgical intervention for the lower extremities in the past year or had a neurological problem were excluded. All participants provided written informed consent prior to participation. The Ethical Committee of Waseda University approved this experiment in accordance with the Helsinki Declaration (Ethics ID: 2015-010).

Bipolar fine-wire intramuscular electrodes (Unique medical Co., Ltd., Japan) were employed for measurement of TP and FDL muscle activity. All electrodes and needles were sterilized in an autoclave prior to insertion. The fine-wire intramuscular electrodes were inserted into the right TP muscle belly. During insertion, the tip of the needle and muscles were observed by using an ultrasound system (LOGIQe, GE, USA). The posterior approach was employed for insertion into the $\mathrm{TP}^{10)}$. In this manner, the fine-wire intramuscular electrodes were also inserted into the right FDL. Accurate insertion into TP and FDL was checked with the muscle activity level during active foot pronation, adduction, and plantar flexion for the TP and toe flexion for the FDL. If the EMG amplitude did not appear for each motion, it was defined as failed insertion and excluded from the data. For the PL measurement, surface electrodes (BlueSensor N-00-S, METS Co., Japan) were placed on the muscle belly of the right PL. The skin was rubbed with an abrasive and alcohol to decrease skin impedance. A wireless EMG telemeter system (BioLog DL-5000, S\&ME Co., Japan) was used to measure both fine-wire and surface EMGs.

Gait tasks were performed under three conditions: (1) barefoot, (2) with footwear, and (3) with footwear plus orthoses (orthoses). All participants wore the same type of footwear (Calcetto Le3, Asics, Japan) and prefabricated orthoses (Athlete grip7, Winning One Inc., Japan). Prefabricated orthoses was employed in this study because it is more clinically meaningful if easy and low cost prefabricated orthoses can change the muscle activity pattern of the calf muscles. The upper layer of the prefabricated insole was made of poly microsuede and the base material was ethyl vinyl acetate. The orthoses had three arch supports for the medial longitudinal, lateral longitudinal, and transverse arches. These arch supports functioned to prevent from lowering the arches. Especially medial longitudinal arch support was expected to control excessive foot pronation.

A force plate (Kistler instrument AG, Switzerland) was placed on the middle of the walkway. The force plate and EMG system were synchronized for simultaneous data collection. In each condition, participants were instructed to walk at comfortable speed on the walkway and step on the force plate with the right foot during gait trials. Prior to measurement, sufficient practice was allowed for the participants to become familiarized with the task. The measurements were implemented until three successful trials were recorded. Maximum voluntary isometric contractions (MVICs) for TP, PL, and FDL were measured after completing the gait tasks in each condition. All MVIC measurements were performed in a sitting position. Over five seconds of MVICs were measured for each muscle. One-second periods of time, including the maximum root mean square (RMS) from the entire MVICs data, were recorded and used as the MVIC values for each muscle.

One stance phase per gait trial, which was defined from the force plate data, was selected to analyze the muscle activity level of each muscle. When the vertical ground force exceeded more than $10 \mathrm{~N}$, the time frame was defined as heel contact. In the same manner, when the vertical ground reaction force decreased less than $10 \mathrm{~N}$, the time frame was defined as toe off. In addition, the stance phase was further divided into contact, midstance, and propulsion phases. The anterior-posterior force value from the force plate data was used to define these phases (Fig. 1). The contact phase was defined from heel contact to the negative peak value of the anterior-posterior force. The midstance was defined from the negative peak value to the time frame when the anterior-posterior force value reached zero. The propulsion phase extended from zero value of the anteriorposterior force to toe off. The sampling rate of EMG and the force plate data was 1,000 Hz. Raw EMG signals were analyzed with software (BIMUTAS-Video, Kissei Comtec Co., Ltd., Japan). The EMG data were rectified, high-pass filtered at $20 \mathrm{~Hz}$, and low-pass filtered at $500 \mathrm{~Hz}$ to remove motion artifacts. Data from the gait tasks was normalized with MVIC values, using a RMS value presenting as \% MVIC. The \% MVIC values from one stance phase from each trial and task were averaged and used for statistical analysis.

In order to confirm the normal distribution of EMG data, Kolmogorov-Smirnov test was employed. Because all data had a normal distribution, one-way repeated measure ANOVAs was conducted to compare the muscle activity level of each muscle in the three conditions during each phase. If Mauchley's test detected a violation of the assumption for sphericity, the $F$-ratio was determined from the Greenhouse-Geisser epsilon. Bonferroni post hoc analysis was used if one-way repeated measures ANOVAs detected significant differences. Partial eta squared was employed to calculate the effect size (ES). Alpha level was set at 0.05 . 


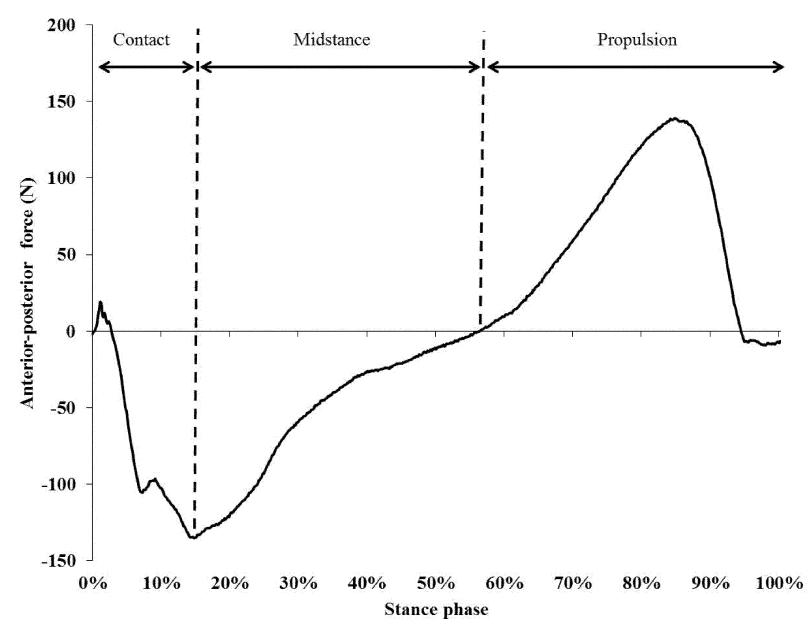

Fig. 1. Definition of the phases based on the anterior-posterior force data during stance phase

The contact phase was defined from the beginning of the stance phase to the negative peak value. The midstance phase was defined from the negative peak value to the zero value. The propulsion phase was defined from the zero value to the end of the stance phase.

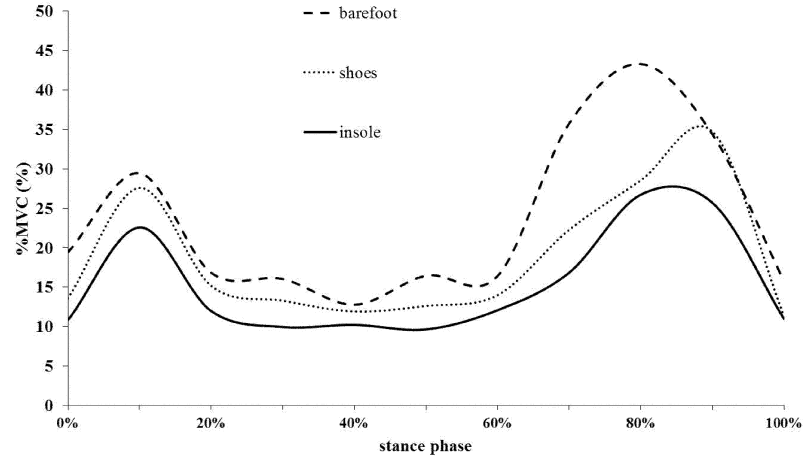

Fig. 2. Waveform of TP muscle activity in each condition during gait

Table 1. \% MVIC comparison among three conditions in each phase

\begin{tabular}{llccc}
\hline \multirow{2}{*}{ Muscle } & Phase & \multicolumn{3}{c}{ Conditions } \\
\cline { 3 - 5 } & & Barefoot & Footwear & Orthoses \\
\hline TP & Total & $32.4 \pm 15.2$ & $26.1 \pm 17.6$ & $21.5 \pm 13.0$ \\
& Contact & $24.6 \pm 20.9$ & $23.3 \pm 20.6$ & $19.7 \pm 14.2$ \\
& Midstance* & $21.8 \pm 13.4$ & $16.3 \pm 10.0$ & $12.3 \pm 7.5$ \\
& Propulsion* & $39.4 \pm 21.0$ & $29.8 \pm 22.1$ & $24.5 \pm 16.6^{* *}$ \\
PL & Total & $31.7 \pm 16.4$ & $30.2 \pm 13.6$ & $30.7 \pm 15.4$ \\
& Contact & $14.4 \pm 6.3$ & $16.7 \pm 8.0$ & $14.5 \pm 8.4$ \\
& Midstance & $22.3 \pm 15.9$ & $22.3 \pm 13.8$ & $21.2 \pm 13.6$ \\
& Propulsion & $38.2 \pm 22.1$ & $36.9 \pm 16.9$ & $38.1 \pm 17.9$ \\
FDL & Total & $35.4 \pm 16.3$ & $39.4 \pm 18.5$ & $40.0 \pm 18.5$ \\
& Contact & $19.7 \pm 11.5$ & $17.5 \pm 7.5$ & $13.7 \pm 5.5$ \\
& Midstance & $26.3 \pm 19.5$ & $23.3 \pm 12.7$ & $20.4 \pm 14.0$ \\
& Propulsion & $47.8 \pm 22.0$ & $52.0 \pm 26.0$ & $53.0 \pm 26.4$ \\
\hline
\end{tabular}

TP: tibialis posterior, PL: peroneus longus, FDL: flexor digitorum longus

*One-way repeated measures ANOVA showed a significant difference $(\mathrm{p}<0.05)$

**Post hoc analysis showed a significant difference between barefoot and orthoses $(\mathrm{p}<0.05)$

\section{RESULTS}

Because of the EMG measurement failure for TP and FDL, the total number of participants decreased to ten (age: $25 \pm$ 5.0 years, height: $167.9 \pm 5.7 \mathrm{~cm}$, weight: $61.5 \pm 7.8 \mathrm{~kg}$ ). Four participants were excluded because of failed electrode insertion into the TP and two for inaccurate placement in the FDL.

The results of the \% MVICs for each muscle in each condition and phase are presented in Table 1. The TP showed a significant difference in the \% MVIC among three conditions in midstance $\left(F_{(2,18)}=4.66, \mathrm{p}=0.023, \mathrm{ES}=0.34\right)$ and propulsion phase $\left(F_{(2,18)}=3.64, \mathrm{p}=0.047, \mathrm{ES}=0.29\right)$. There was no significant difference in the total stance and contact phase $(\mathrm{p}>0.05)$. 
Bonferroni post hoc analysis revealed that the orthoses condition showed significantly less \% MVIC value of TP compared to barefoot condition in the propulsion phase alone $(\mathrm{p}<0.036)$. Although a significant difference was detected by one-way repeated measures ANOVAs in midstance phase, post hoc analysis did not show a significant difference among three conditions ( $p>0.05$ ). The average waveform of TP during the stance phase of walking is shown in Fig. 2. The \% MVIC of the FDL and PL showed that there were no statistical differences between the three conditions in each phase $(p>0.05)($ Table 1).

\section{DISCUSSION}

The current study is the first study that assessed the effect of the orthoses on EMG amplitude change of TP, PL, and FDL simultaneously during gait. The current study revealed that the orthoses significantly reduced muscle activity level of TP in midstance and propulsion phases during gait. In contrast, muscle activity level of the PL and FDL remained the same for all conditions in each phase.

The muscle activity pattern of TP during gait displayed a biphasic EMG waveform. This waveform is similar to previous studies $^{11)}$. The function of TP during gait is to prevent excessive foot pronation during the contact phase and to increase the stiffness of foot during the propulsion phase ${ }^{4,11}$. In addition, the medial longitudinal arch is maintained by TP during weight bearing activities ${ }^{12}$. Owing to the relative distance between the axes of the subtalar joint and TP tendon, TP functions as the strongest supinator muscle of the hindfoot ${ }^{5,13)}$. Foot supination in the propulsion phase is fundamental in increasing rigidity of the foot, a function known as midtarsal locking mechanism that has an advantage of improving force transmission efficiency ${ }^{14)}$. Considering this reduction of the muscle activity level of TP in the propulsion phase with orthoses, the rearfoot might be stabilized, and the function of TP, which increase the stiffness of the foot, can be compensated by the orthoses. However, this result is inconsistent with a previous study. The results of the previous study reported that a significant reduction in the RMS amplitude of TP was found during the contact phase of gait with prefabricated and customized foot orthoses compared to shoes only condition ${ }^{7}$. In the previous study, there were no significant differences in RMS amplitude during midstance/propulsion phase among the conditions. Because the previous study divided the contact phase into 2 phases; contact phase and midstance/propulsion phase based on the EMG pattern, number of phases and hence the definition of phases are different from the current study. These differences might have led to the inconsistent results. Although the phase where the reduction of TP muscle activity found was different, orthoses can decrease the muscle activity level of TP while walking.

PL generates eversion torque to the subtalar joint ${ }^{13}$. Contraction of PL during gait results in decreased foot rigidity and increased energy dissipation rate ${ }^{4}$. These mechanisms are antagonistic in function with TP and are important for shock attenuation. The effect of orthoses on PL activity has been described by several studies ${ }^{8,15,16)}$. The results of these previous studies were diverse. Two studies showed that significantly higher muscle activity level was observed with orthoses ${ }^{8,15)}$. However, a previous study reported that muscle activity level of the PL did not alter ${ }^{15}$. Significant increase of the PL muscle activity level was considered due to laterally unstable conditions caused by posting. In order to stabilize the subtalar joint against the tilting, the PL needs further contraction. In the current study, no wedge was added to the orthoses. This may influence the result that orthoses did not affect the muscle activity level of PL.

The muscle activity of FDL also showed no difference in the three conditions in all phases. Because no previous study assessed the FDL activity change with orthoses, the results cannot be compared to those of other researches. In normal gait, the FDL contracts from midstance to toe off during gait ${ }^{17)}$. Similar to TP, FDL isometrically contracts to maintain the height of the longitudinal arch of the foot thereby improving the propulsion force transmission efficacy ${ }^{6}$. Although orthoses might be able to support medial and lateral longitudinal arches as well as transverse arch, it might not be sufficient to support arches and compensate for the activity of FDL.

Clinically, the reduction of the muscle activity level of TP might be beneficial for some injuries. The traction force applied to the medial distal tibia is one of the mechanisms resulting in medial tibial stress syndrome (MTSS). Recent studies demonstrated that TP does not have direct insertion into the symptomatic location of the medial distal tibia ${ }^{18)}$, the TP, FDL, and soleus muscles are responsible for generating the traction force on the medial distal tibia via the crural fascia ${ }^{19)}$. Consequently, the reduction of TP muscle activity with orthoses can be beneficial for patients who have MTSS.

The small number of subjects is the limitation of this study. The sample size decreased due to technical errors in measuring the fine-wire EMG. Precise insertion of the electrodes into the muscle belly of TP and FDL is technically challenging and must be carefully performed to avoid damaging these structures ${ }^{10)}$. Additionally, muscle contraction of the calf muscles pulls out the wires even when the tips are properly inserted. More stable and accurate insertion technique should be considered for future studies.

In summary, the current study assessed the muscle activity change of the TP, FDL, and PL in three conditions, barefoot, footwear, and orthoses, during gait. Gait with orthoses exhibited significantly less TP muscle activity than that in the barefoot condition. This significant difference was found in midstance and propulsion phases. PL and FDL had no significant muscle activity changes in the three conditions in all phases. Reduction of TP muscle activity might be beneficial for patients who have excessive TP contraction related injuries, such as MTSS. Further research is required to clarify the clinical effect of orthoses in patients with MTSS. 


\section{REFERENCES}

1) Mills K, Blanch P, Chapman AR, et al.: Foot orthoses and gait: a systematic review and meta-analysis of literature pertaining to potential mechanisms. Br J Sports Med, 2010, 44: 1035-1046. [Medline] [CrossRef]

2) McMillan A, Payne C: Effect of foot orthoses on lower extremity kinetics during running: a systematic literature review. J Foot Ankle Res, 2008, 1: 13. [Medline] [CrossRef]

3) MacLean C, Davis IM, Hamill J: Influence of a custom foot orthotic intervention on lower extremity dynamics in healthy runners. Clin Biomech (Bristol, Avon), 2006, 21: 623-630. [Medline] [CrossRef]

4) Kokubo T, Hashimoto T, Nagura T, et al.: Effect of the posterior tibial and peroneal longus on the mechanical properties of the foot arch. Foot Ankle Int, 2012, 33: 320-325. [Medline] [CrossRef]

5) Semple R, Murley GS, Woodburn J, et al.: Tibialis posterior in health and disease: a review of structure and function with specific reference to electromyographic studies. J Foot Ankle Res, 2009, 2: 24. [Medline] [CrossRef]

6) Hofmann CL, Okita N, Sharkey NA: Experimental evidence supporting isometric functioning of the extrinsic toe flexors during gait. Clin Biomech (Bristol, Avon), 2013, 28: 686-691. [Medline] [CrossRef]

7) Murley GS, Landorf KB, Menz HB: Do foot orthoses change lower limb muscle activity in flat-arched feet towards a pattern observed in normal-arched feet? Clin Biomech (Bristol, Avon), 2010, 25: 728-736. [Medline] [CrossRef]

8) Murley GS, Bird AR: The effect of three levels of foot orthotic wedging on the surface electromyographic activity of selected lower limb muscles during gait. Clin Biomech (Bristol, Avon), 2006, 21: 1074-1080. [Medline] [CrossRef]

9) Murley GS, Landorf KB, Menz HB, et al.: Effect of foot posture, foot orthoses and footwear on lower limb muscle activity during walking and running: a systematic review. Gait Posture, 2009, 29: 172-187. [Medline] [CrossRef]

10) Won SJ, Kim JY, Yoon JS, et al.: Ultrasonographic evaluation of needle electromyography insertion into the tibialis posterior using a posterior approach. Arch Phys Med Rehabil, 2011, 92: 1921-1923. [Medline] [CrossRef]

11) Murley GS, Buldt AK, Trump PJ, et al.: Tibialis posterior EMG activity during barefoot walking in people with neutral foot posture. J Electromyogr Kinesiol, 2009, 19: e69-e77. [Medline] [CrossRef]

12) Kamiya T, Uchiyama E, Watanabe K, et al.: Dynamic effect of the tibialis posterior muscle on the arch of the foot during cyclic axial loading. Clin Biomech (Bristol, Avon), 2012, 27: 962-966. [Medline] [CrossRef]

13) Klein P, Mattys S, Rooze M: Moment arm length variations of selected muscles acting on talocrural and subtalar joints during movement: an in vitro study. J Biomech, 1996, 29: 21-30. [Medline] [CrossRef]

14) Okita N, Meyers SA, Challis JH, et al.: Midtarsal joint locking: new perspectives on an old paradigm. J Orthop Res, 2014, 32: 110-115. [Medline] [CrossRef]

15) Mündermann A, Wakeling JM, Nigg BM, et al.: Foot orthoses affect frequency components of muscle activity in the lower extremity. Gait Posture, 2006, 23: 295-302. [Medline] [CrossRef]

16) Tomaro J, Burdett RG: The effects of foot orthotics on the EMG activity of selected leg muscles during gait. J Orthop Sports Phys Ther, 1993 , 18 : $532-536$. [Medline] [CrossRef]

17) Grant AD: Gait analysis: normal and pathological function. JAMA, 2010, 304: 907. [CrossRef]

18) Stickley CD, Hetzler RK, Kimura IF, et al.: Crural fascia and muscle origins related to medial tibial stress syndrome symptom location. Med Sci Sports Exerc, 2009, 41: 1991-1996. [Medline] [CrossRef]

19) Bouché RT, Johnson CH: Medial tibial stress syndrome (tibial fasciitis): a proposed pathomechanical model involving fascial traction. J Am Podiatr Med Assoc, 2007, 97: 31-36. [Medline] [CrossRef] 